\title{
ARTICLE
}

\section{Designing quantum dots for solotronics}

\author{
J. Kobak ${ }^{1, \star}$, T. Smoleński ${ }^{1, \star}$, M. Goryca ${ }^{1}$, M. Papaj ${ }^{1}$, K. Gietka ${ }^{1}$, A. Bogucki1, M. Koperski ${ }^{1}$, J.-G. Rousset ${ }^{1}$, \\ J. Suffczyński ${ }^{1}$ E. Janik ${ }^{1}$, M. Nawrocki ${ }^{1}$, A. Golnik${ }^{1}$, P. Kossacki ${ }^{1} \&$ W. Pacuski ${ }^{1}$
}

Solotronics, optoelectronics based on solitary dopants, is an emerging field of research and technology reaching the ultimate limit of miniaturization. It aims at exploiting quantum properties of individual ions or defects embedded in a semiconductor matrix. It has already been shown that optical control of a magnetic ion spin is feasible using the carriers confined in a quantum dot. However, a serious obstacle was the quenching of the exciton luminescence by magnetic impurities. Here we show, by photoluminescence studies on thus-far-unexplored individual $\mathrm{CdTe}$ dots with a single cobalt ion and CdSe dots with a single manganese ion, that even if energetically allowed, nonradiative exciton recombination through single-magnetic-ion intra-ionic transitions is negligible in such zero-dimensional structures. This opens solotronics for a wide range of as yet unconsidered systems. On the basis of results of our single-spin relaxation experiments and on the material trends, we identify optimal magnetic-ion quantum dot systems for implementation of a single-ion-based spin memory.

\footnotetext{
${ }^{1}$ Institute of Experimental Physics, Faculty of Physics, University of Warsaw, Hoża 69, Warsaw 00-681, Poland. * These authors contributed equally to this work. Correspondence and requests for materials should be addressed to W.P. (email: Wojciech.Pacuski@fuw.edu.pl).
} 
$\mathrm{T}$ he term solotronics ${ }^{1}$ has been introduced to describe recent advances ${ }^{2,3}$ in fabricating and operating semiconductor optoelectronic devices based on single dopants or defects for applications in computer memories, quantum computation and on-demand photon sources. The most advanced solotronics technology has been developed for nitrogen-vacancy $(\mathrm{N}-\mathrm{V})$ defect centres in diamond, for which it has been shown that quantum states can be prepared and read out and spin can be manipulated using microwave and optical transitions ${ }^{4-7}$. Defects similar to $\mathrm{N}-\mathrm{V}$ centres have also been observed in semiconductors ${ }^{8}$ such as $\mathrm{SiC}^{9} . \mathrm{SiC}$ is more compatible with present semiconductor-based technology than diamond; however, owing to the weak coupling of free carriers to defect centres, it does not allow for electrically controlled operation. More promising in this view is another solotronic system: a single magnetic ion embedded in a semiconductor quantum dot $(\mathrm{QD})^{10-12}$. Here, the spin state of the single ion can be prepared and manipulated both electrically ${ }^{13,14}$ and optically ${ }^{12,15,16}$ through injection of spinpolarized carriers. The $s, p-d$ exchange coupling between the magnetic ion and the band carrier enables an unambiguous readout of the spin projection of the ion from the energy and polarization of a photon emitted by the $\mathrm{QD}^{10,11}$. The ease of optical addressing of individual QDs enables operation on the level of single ions ${ }^{12,15}$. Multiple magnetic ions can be coupled by carriers in one $\mathrm{QD}^{17-19}$ or by QDs coupling through tunnelling carriers $^{12}$ or photonic structures ${ }^{20}$. The use of semiconductor heterostructures opens a huge area for testing new ideas for single-ion spin operation, as it offers a band gap and strain engineering, tuning energies of optical and microwave transitions, Fermi level manipulation and integration with $\mathrm{p}-\mathrm{i}-\mathrm{n}$ structures.

A severe limitation of QDs doped with transition metal ions was attributed to the efficient recombination channel introduced by magnetic ion $s^{21-29}$ when the exciton energy is higher than the intra-ionic transition energy, which should result in quenching of exciton emission. Therefore, the only QD systems with singlemagnetic ions considered so far were those where the intra-ionic transition energies exceed the exciton energy, namely $\mathrm{Mn}^{2+}$ embedded in CdTe/ZnTe and InAs/GaAs QDs ${ }^{10-16,30-32}$. On the other hand, incorporation of magnetic ions such as $\mathrm{Cr}, \mathrm{Fe}, \mathrm{Co}, \mathrm{Ni}$ or $\mathrm{Cu}$ would bring physical properties like orbital momentum, reduced number of spin states, sensitivity to local strain, the Jahn-Teller effect or isotopes with zero nuclear spin, offering additional degrees of freedom for designing quantum states. Extending the studies of single magnetic ions to other QD systems such as $\mathrm{CdSe}, \mathrm{ZnSe}, \mathrm{CdS}, \mathrm{ZnS}, \mathrm{ZnO}, \mathrm{GaN}$ or other wide-gap semiconductors would offer, in turn, increased photoluminescence (PL) efficiency at higher temperatures, enhancement of the exchange interaction within excitons and between excitons and ions or reduction of spin-orbit coupling and the resulting spin relaxation rates.

We report here for the first time on a single cobalt ion in a $\mathrm{CdTe} / \mathrm{ZnTe} \mathrm{QD}$ and a single manganese ion in a CdSe/ZnSe QD. The spin states of the dopant ions are mapped onto the QD optical transitions recorded in a magneto-PL measurement. We employ PL decay measurements to demonstrate that contrary to the case of systems with many magnetic ions ${ }^{21-27}$, the exciton emission quenching is negligible for single dopants. Through modulated, polarization-resolved PL measurements we access the single-spin relaxation and prove that all-optical control of a single magnetic moment is feasible in the systems studied. Moreover, we show that spin properties of magnetic QDs can be designed by an independent choice of the magnetic ion and the QD material. We discuss the role of the electronic configuration of $d$-shell, spin-orbit and hyperfine interactions and, finally, we indicate the most promising design of future QD-based solotronic systems.

\section{Results}

PL spectra of QDs with single magnetic ions. Samples with selfassembled QDs containing magnetic ions are grown by molecular beam epitaxy (see Methods) and studied by PL at low temperatures (down to $1.5 \mathrm{~K}$ ). The use of a microscope objective enables the observation of exciton emission lines of individual QDs. Among sharp lines with a typical emission pattern of nonmagnetic QDs ${ }^{33}$ (majority) and broader lines related to QDs with many magnetic ions ${ }^{19,22,34}$, it is possible to identify the emission multiplets characteristic of individual QDs with exactly one magnetic ion, where the ion spin state is probed by confined excitonic complexes (neutral and charged excitons, biexciton and so on). In particular, the emission related to a bright state of a neutral exciton (Fig. 1) consists of a set of lines split by the $s, p-d$ exchange interaction. The number of lines is determined by the possible magnetic ion spin projections on the growth axis, this being the exciton quantization axis. More specifically, for $\mathrm{Mn}^{2+}$ with spin $5 / 2$ there are six spin projections: $\pm 5 / 2, \pm 3 / 2$ and $\pm 1 / 2$, so we observe six lines for a CdSe QD with a single $\mathrm{Mn}^{2+}$ ion (Fig. 1a,b,c), analogously to a CdTe QD with the same ion $^{10}$. The $\mathrm{Co}^{2+}$ ion spin is $3 / 2$ and thus there are four spin projections: $\pm 3 / 2$ and $\pm 1 / 2$, resulting in four lines for a CdTe QD with a single $\mathrm{Co}^{2+}$ ion (Fig. 1d-f). However, the intensity of the lines related to $\mathrm{Co}^{2+}$ spin projections $\pm 3 / 2$ (outer lines) can be significantly different from those related to the spin projections $\pm 1 / 2$ (inner lines). The $\mathrm{Co}^{2+}$ ion orbital momentum is nonzero, and $\mathrm{Co}^{2+}$ incorporated in the crystal is very sensitive to local anisotropy and strain, which lead to the splitting of $\pm 3 / 2$ and $\pm 1 / 2$ states and a difference in their occupancy ${ }^{35-37}$. The QD shown in Fig. 1d exhibits outer lines more intense than the inner lines. This means that in this case the strain makes the state with spin $\pm 3 / 2$ the ground state. The difference between the spin state occupancies (and therefore the exciton line intensities) is more pronounced at low temperatures, as expected from Boltzmann statistics.

The identification of the excitonic lines is confirmed by the analysis of PL spectra measured as a function of the magnetic field (Fig. 2). Zeeman shifts of bright and dark excitonic transitions for a CdSe QD with $\mathrm{Mn}^{2+}$ (Fig. 2a) can be well described (Fig. 2b) using the model proposed for a CdTe QD with $\mathrm{Mn}^{2+}$ (ref. 10). In order to account for all the observed features of the PL spectrum from a CdTe QD with a single $\mathrm{Co}^{2+}$ (Fig. 2c,d), we extend the model by introducing a strain vector that induces a zero-field splitting of the $\mathrm{Co}^{2+}$ spin states (see Methods). Figure 2e shows the scheme of excitonic optical transitions for a relatively simple case, when the strain-induced $\mathrm{Co}^{2+}$ anisotropy axis is parallel to the growth axis. In plane anisotropy of $\mathrm{Co}^{2+}$ would induce an additional excitonic mixing, analogous to the case of a neutral Mn centre $\left(d^{5}+h\right)$ in a InAs/ GaAs QD ${ }^{31}$. Typical PL spectra of various QDs with singlemagnetic ions are presented in Supplementary Note 1 and Supplementary Figs 1 and 2.

Recombination channels for excitons. Quenching of exciton PL by a recombination channel introduced by a magnetic dopant is an important obstacle in studying diluted magnetic semiconductors (DMS) with an energy gap larger than the magnetic ions' internal transition energies ${ }^{25,26,28}$. This is the case for DMS with magnetic ions other than $\mathrm{Mn}$ (for example, $\mathrm{V}, \mathrm{Cr}, \mathrm{Fe}, \mathrm{Co}, \mathrm{Ni}$ and $\mathrm{Cu})^{36,38,39}$. Only $\mathrm{Mn}^{2+}$ ions have relatively large intra-ionic transition energies (about $2.2 \mathrm{eV}$ ) allowing for efficient PL studies of excitons in $\mathrm{Cd}_{1-x} \mathrm{Mn}_{x} \mathrm{Te}^{40,41}$ (energy gap $E_{\mathrm{g}}(\mathrm{CdTe})=1.6 \mathrm{eV}$ ) and $\mathrm{Ga}_{1-} \mathrm{Mn}_{x}$ As with low Mn concentration $(x \leqslant 0.13 \%)^{42,43}$ (energy gap $E_{\mathrm{g}}(\mathrm{GaAs})=1.5 \mathrm{eV}$ ). As a consequence, the first approaches to studies of dots with single magnetic ions were 

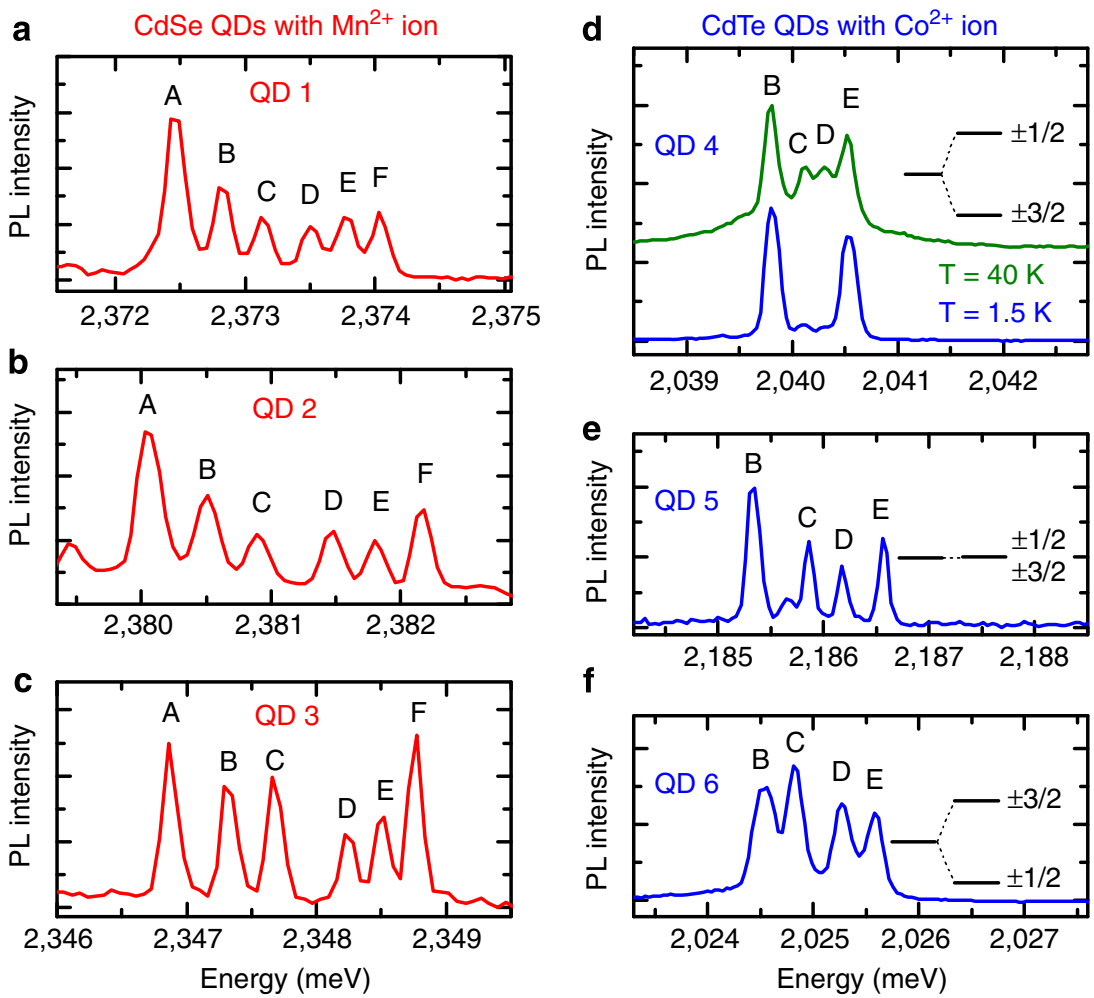

Figure 1 | Excitonic PL spectra of quantum dots with single magnetic ions. (a-c) PL spectra of CdSe QDs with single Mn ${ }^{2+}$ ions. (d-f) PL spectra of CdTe QDs with single $\mathrm{Co}^{2+}$ ions. Spectra are measured at zero magnetic field and low temperature, $T=1.5 \mathrm{~K}$. The exciton splitting results from the $s, p-d$ exchange interaction with a magnetic ion. For $\mathrm{QD}$ with $\mathrm{Mn}^{2+}$ ion $(S=5 / 2)$, we observe six components that in $\sigma^{ \pm}$circular polarization correspond to $\mathrm{Mn}^{2+}$ spin projections $\mp 5 / 2(A), \mp 3 / 2(B), \mp 1 / 2(C), \pm 1 / 2(D), \pm 3 / 2(E)$ and $\pm 5 / 2(F)$ while for QD with $\mathrm{Co}^{2+}$ ion $(S=3 / 2)$, there are four components related to $\mathrm{Co}^{2+}$ spin projections $\mp 3 / 2(B), \mp 1 / 2(C), \pm 1 / 2(D)$ and $\pm 3 / 2$ (E). Intensities of various lines are related to a strain and resulting occupancy of magnetic ion states. For $\mathrm{Mn}^{2+}$, the effect of the strain is negligible (a-c) while for $\mathrm{Co}^{2+}$ the impact is visible (d-f), for example, cobalt shown in (d) has a fundamental state with spin $\pm 3 / 2$, so observation of $\pm 1 / 2$ states requires an increase in temperature. The upper curve in (d) was blueshifted by $4.42 \mathrm{meV}$ in order to compensate for the temperature shift.

limited to manganese and QDs with low emission energy: CdTe/ $\mathrm{ZnTe}^{10}$ and InAs/GaAs ${ }^{11}$. The relatively small energy gap of CdSe $\left(E_{\mathrm{g}}(\mathrm{CdSe})=1.7 \mathrm{eV}\right)$ results in energy transfer from the magnetic

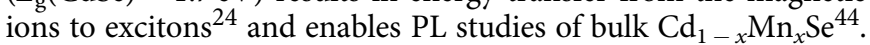
In the case of nanostructures, quantum confinement increases the exciton energy. As a consequence, the direction of the energy transfer is reversed, leading to PL quenching $22-25,27$ and a significant shortening of the exciton lifetime $e^{21,28}$.

However, our measurements of the PL decay performed on the $\mathrm{CdTe} / \mathrm{ZnTe}$ sample reveal the same lifetime of about $220 \pm 40 \mathrm{ps}$ for a $\mathrm{QD}$ with and without a single $\mathrm{Co}^{2+}$ ion (see Fig. $3 \mathrm{c}, \mathrm{d}$ ). Also, for selenide QDs exciton lifetime is measured to be around $220 \pm 40 \mathrm{ps}$ for a dot with and without a single $\mathrm{Mn}^{2+}$ (Fig. 3e,f). Therefore, we do not observe shortening of the exciton lifetime induced by single magnetic ions. Moreover, the PL intensity of individual QDs with single magnetic ions is comparable to that of nonmagnetic QDs. The above findings indicate that the quenching of excitonic emission is not efficient when single dopants are introduced to QDs. We interpret this as the result of the discrete density of states of zero-dimensional systems with exactly one magnetic ion. In this case the exciton energy cannot be efficiently transferred either to phonons or to the magnetic ion owing to an energy mismatch. This effect can be compared with the phonon bottleneck that can hinder exciton relaxation to the ground state of zero-dimensional systems ${ }^{45,46}$ if other relaxation channels, such as the Auger process ${ }^{47-49}$, are not efficient. Furthermore, energy transfer from exciton to magnetic ions can also be hindered by spin conservation selection rules ${ }^{50-52}$.
The high efficiency of radiative exciton recombination found in the present work is different with respect to the bulk DMS case, where the exciton is typically coupled to an ensemble of magnetic ions, able to absorb energy over a wide range, for example, by a collective change of spin configuration. The above results not only show that PL studies of DMS can be significantly extended by using zero-dimensional structures, but they also imply highfidelity optical readout of a single-dopant quantum state in a QD.

Spin relaxation of a single magnetic ion. In order to investigate the spin relaxation dynamics of a $\mathrm{Mn}^{2+}$ ion embedded in a $\mathrm{CdSe} / \mathrm{ZnSe} \mathrm{QD}$, we measure time-resolved PL in a magnetic field, with an on-/off-modulated non-resonant laser excitation (Fig. 4). When the laser is switched on, the optically created excitons injected into the QD depolarize the spin of the embedded magnetic ion (typically within several hundreds of nanoseconds). Then the laser is switched off for a dark period, during which the $\mathrm{Mn}^{2+}$ spin approaches the state of alignment to the magnetic field direction. Finally, the laser is switched on again to perform the readout of the spin state by measuring the temporal increase of the PL amplitude of a high-energy line. This line corresponds to the state of the $\mathrm{Mn}^{2+}$ ion with spin oriented parallel to the magnetic field. Figure $4 \mathrm{~b}, \mathrm{c}$ shows the dependence of the PL amplitude on the length of the dark period for two different values of magnetic field. The exponential fits allow us to determine the $\mathrm{Mn}^{2+}$ ion spin relaxation times in a CdSe QD at helium temperature, in magnetic fields of $4 \mathrm{~T}$ and $8 \mathrm{~T}$ to be equal to 
a

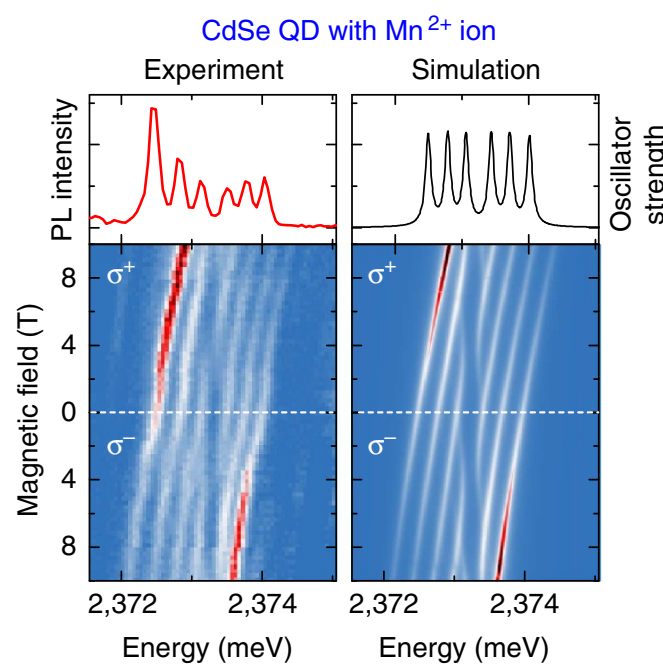

C

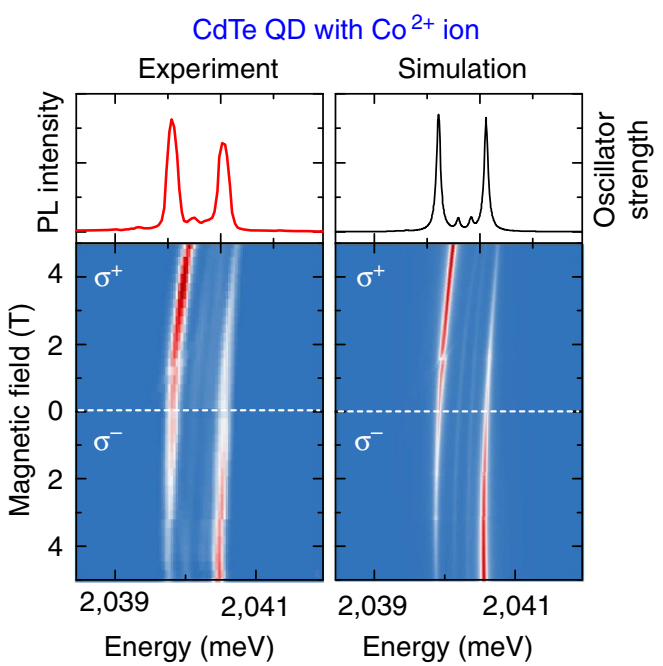

e

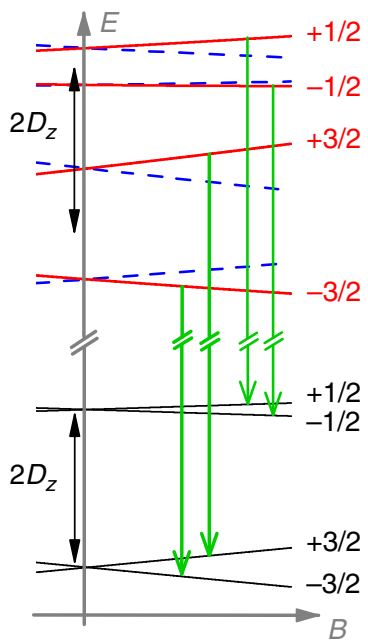

Figure 2 | Magneto-optical spectroscopy of QDs with single magnetic ions. $T=1.5 \mathrm{~K}$. PL of an exciton in a $\mathrm{CdSe} Q \mathrm{QD}$ with a $\mathrm{Mn}{ }^{2}+$ ion as a function of magnetic field in Faraday configuration (a) and a corresponding simulation (b). An analogous experiment (c) and simulation (d) for a CdTe QD with $\mathrm{Co}^{2+}$. More intense low-energy lines in the polarization $\sigma^{+}$and high-energy lines in the polarization $\sigma^{-}$at high a magnetic field indicate alignment of ion spins along the external magnetic field direction. Parameters used for calculation of (b) and (d) are listed in Supplementary Table 1. The scheme of excitonic transitions (e) in $\sigma^{+}$polarization is shown for a QD with $\mathrm{Co}^{2+}$ for a relatively simple case, where the strain-induced $\mathrm{Co}^{2+}$ anisotropy axis is parallel to the growth axis and the resulting zero-field spin splitting of $\mathrm{Co}^{2+}$ states is equal to $2 D_{z}$, which is in the microwave range, of the order of $1 \mathrm{meV}$. The spin projection of $\mathrm{Co}^{2+}$ is indicated.

a

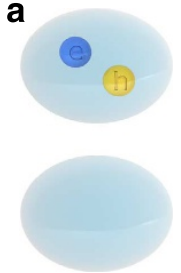

b

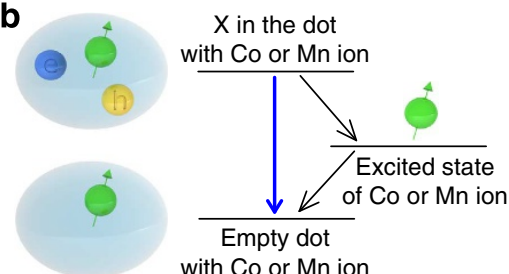

$X$ in the dot

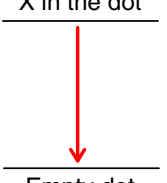

with $\mathrm{Co}$ or $\mathrm{Mn}$ ion
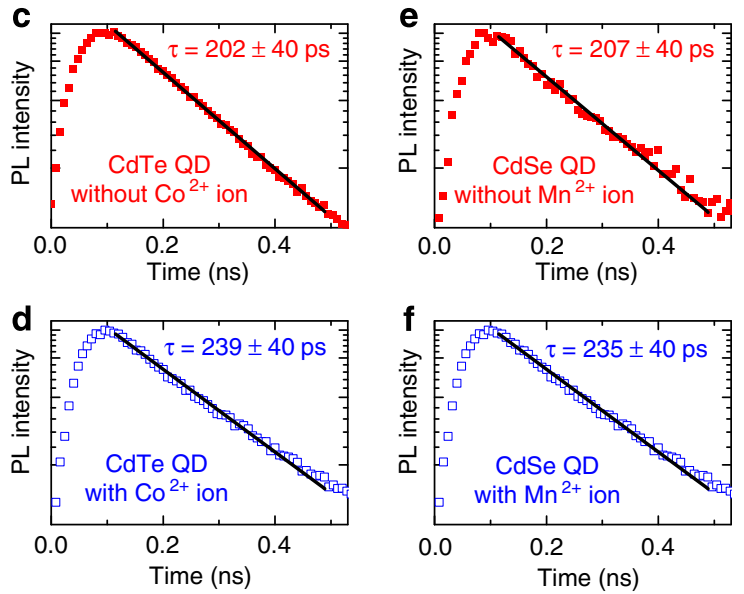

Figure 3 | Exciton recombination channels in a QD with a single magnetic ion. (a) Radiative channel in a nonmagnetic QD, (b) radiative and nonradiative channels in a QD with a magnetic ion, which exhibits intra-ionic transitions at energies lower than the exciton energy. Exciton PL decay measurement for a CdTe QD (c), CdTe QD with a single $\mathrm{Co}^{2+}(\mathbf{d}), \mathrm{CdSe} \mathrm{QD}(\mathbf{e})$ and CdSe QD with a single $\mathrm{Mn}^{2+}$ (f). We do not observe any impact of single dopants on exciton decay time $(\tau)$, which implies that the nonradiative channel is slower than the radiative channel. Temporal resolution of the set-up is $40 \mathrm{ps}$. Data are collected at zero magnetic field and low temperature, $T=1.6 \mathrm{~K}$.

$135 \mu \mathrm{s}$ and $24 \mu \mathrm{s}$, respectively. It is over an order of magnitude longer than the relaxation time of the $\mathrm{Mn}^{2}+$ ion in a CdTe QD, that is $5 \mu \mathrm{s}$ for $B=4 \mathrm{~T}$ measured by Goryca et al. (private communication, 2013) with the technique reported in ref. 12. Such results demonstrate that modification of the QD material can significantly increase the storage time of information written on the $\mathrm{Mn}^{2+}$ spin, which can noticeably improve the features of quantum memories based on QDs with single magnetic ions. On the other hand, embedding an ion with a different electronic configuration may lead to an acceleration of the spin dynamics. In particular, similar measurements to those described in Fig. 4 performed on a CdTe QD with a single $\mathrm{Co}^{2+}$ ion yield a relaxation time of about $2 \mu$ s in a magnetic field of $3 \mathrm{~T}$, shorter than for a $\mathrm{Mn}^{2+}$ ion in a CdTe QD.
The method of single-spin relaxation measurement based on initial depolarization of magnetic ions presented here has the advantage of being applicable to any QD system with magnetic ions, independently of the choice of photoexcitation energy. However, for particular systems, measurements can be improved by the identification of resonant excitation channels, which allow for efficient transfer of polarization to QDs, and consequently optical polarization of the magnetic ions. Such resonant excitation channels have been identified for (Ga,Mn)As-based quantum wells $^{43}$, for CdTe QDs with single $\mathrm{Mn}$ ions ${ }^{12,15,53}$ and for InAs/GaAs QDs with a single $\mathrm{Mn}^{16}$. For CdSe/ZnSe QDs even quasi resonant excitation results in some degree of $\mathrm{QD}$ exciton polarization ${ }^{54}$. This effect and identification of resonant excitation channels for a CdSe QD with a single Mn will result 

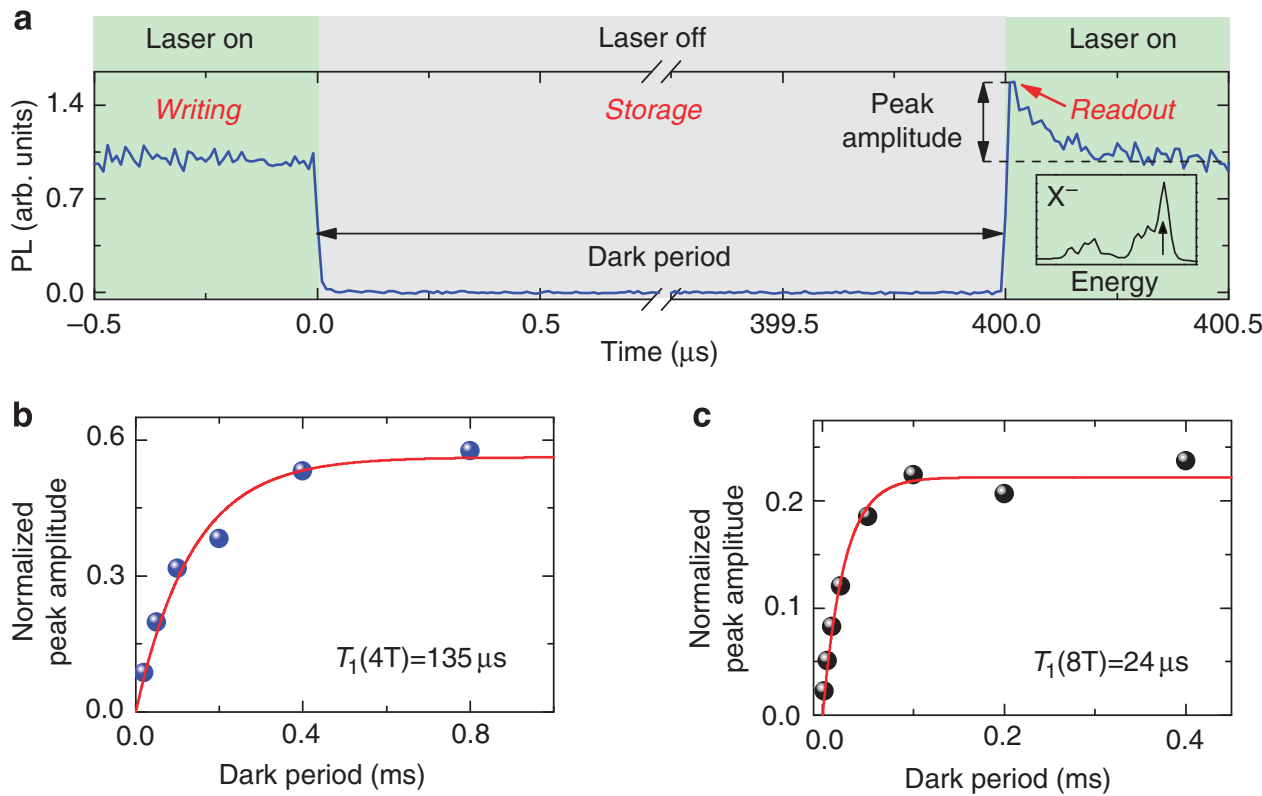

Figure 4 | Relaxation dynamics of a single $\mathbf{M n}^{2+}$ ion spin in a CdSe/ZnSe QD. $T=1.6 \mathrm{~K}$. (a) Temporal profile of the PL intensity of the highest energy $X^{-}$emission line (indicated in the inset) in magnetic field $B=4 \mathrm{~T}$ (detection in $\sigma^{-}$polarization). The nonresonant (405 nm) continuous-wave excitation is subsequently turned on and off for controlled periods of time. The amplitude of the PL intensity peak observed immediately after turning the excitation on indicates the loss of information stored on the $\mathrm{Mn}^{2}+$ spin. $(\mathbf{b}, \mathbf{c})$ The amplitude of the PL intensity peak measured just after turning on the excitation versus length of the dark period for external magnetic fields of $4 \mathrm{~T}$ and $8 \mathrm{~T}$, respectively. The fitted exponential curves yield the storage times $T_{1}$ of information on the $\mathrm{Mn}^{2+}$ ion spin.

in efficient optical orientation of $\mathrm{Mn}$ and will be an important step towards observation of coherent phenomena.

\section{Discussion}

The results presented above highlight the opportunity for extension of QD-based solotronics research to as yet unexplored combinations of semiconductor systems and magnetic ions. In this section, we discuss the optimal systems from the point of view of practical implementation of QD-based solotronic devices. Materials where ion-carrier exchange interaction has been experimentally observed are summarized in Table 1a. Table $1 \mathrm{~b}$ presents the most interesting combinations of QDs and magnetic ions. So far, only two systems have been studied (single Mn in $\mathrm{CdTe} / \mathrm{ZnTe}$ and InAs/GaAs QDs). With our work we introduce a new magnetic ion $-\mathrm{Co}^{2+}$ and a new solotronic system-CdSe/ZnSe.

We note first that for applications in solotronics it is important to lenghten the single-spin relaxation time using magnetic ions without orbital momentum, that is, with a half-filled $d$-shell $\left(d^{5}\right)$. At non-zero magnetic fields, the $\mathrm{Mn}^{2+}$ ions $\left(d^{5}\right)$ in a bulk matrix exhibit a relaxation time longer by two orders of magnitude than for other $3 d$ transition metals ${ }^{55}$. This trend is confirmed in our work for the ions in QDs, since $\mathrm{Co}^{2+}\left(d^{7}\right.$ shell) exhibits a shorter relaxation time than $\mathrm{Mn}^{2+}$. However, since increasing magnetic field enhances spin relaxation ${ }^{55}$ (see Fig. 4), practical applications of solotronic devices will be realized at zero or low magnetic fields. At zero field, the nuclear spin ${ }^{12,15,32,41,56}$ plays the most important role and we predict that another $d^{5}$ ion, $\mathrm{Fe}^{3+}$ with zero nuclear spin, should be more stable than $\mathrm{Mn}^{2}+$ with $5 / 2$ nuclear spin. With $\mathrm{Fe}^{3+}$ and a QD built from isotopically purified group II and VI elements (which are attainable), it will be possible to have a whole system free of nuclear spin and to obtain an extremely long spin relaxation time, as was shown for $\mathrm{N}-\mathrm{V}$ centres in isotopically engineered diamond ${ }^{5}$.

The rate of spin relaxation of isolated ions increases with the strength of the spin-orbit interaction ${ }^{40,55,56}$, which depends on the magnetic ion and the host material ${ }^{57}$. Therefore it is reasonable to use QDs based on semiconductors with light anions: sulphides, oxides and nitrides, as they exhibit a weak spin-orbit interaction. This trend ${ }^{58}$ is confirmed by our results on QDs, as single $\mathrm{Mn}^{2}+$ has a longer relaxation time when it is embedded in a selenide (Fig. 4) than in a telluride ${ }^{12}$ (heavier) (Goryca et al. private communication, 2013) system. For ensembles of isolated $\mathrm{Fe}^{3+}$ and $\mathrm{Mn}^{2+}$ in oxides and sulphides, long spin relaxation times up to $0.4 \mathrm{~s}^{59,60}$ and a spin coherence time of $0.9 \mu \mathrm{s}^{61}$ were demonstrated at helium temperatures.

Manipulation of the stored spin is equally important for application as a long spin relaxation time. Manipulation through the ion-carrier exchange interaction ${ }^{12}$ or microwave radiation ${ }^{61}$ should be feasible for all the systems presented in Table 1b. Another, thus-far-unexploited possibility for single-magnetic-ion spin manipulation in QDs, is related to intra-ionic transitions, which are similar to transitions exploited for defect centres ${ }^{8}$. The intra-ionic transitions could be useful for reading, manipulation and writing of the magnetic ion spin. Spin readout from sharp intra-ionic transitions of $\mathrm{Co}^{2+}$ ions in $\mathrm{Zn}_{1-x} \mathrm{Co}_{x} \mathrm{O}$ layers has been demonstrated in ref. 37 .

It is worth noting that strain gives the possibility of inducing a temporal evolution of spin, tuning the zero-field splitting and reducing the spin degeneracy of the ground state. Thus, it can be profitable for manipulation of ion spins. The effect of strain on $\mathrm{Co}^{2+}$ spin states is shown in Fig. 2e. In order to use strain or crystal field effects, it is desirable to control the anisotropy axis, for example, by using wurtzite structure compounds, where the $c$ axis is expected to define the quantization axis of the zero-field spin splitting. If one wishes, in turn, to eliminate a strain-induced complexity, lattice-matched materials, such as GaAs and AlAs for growth of magnetic QDs by droplet epitaxy, are recommended.

In conclusion, the optical properties of a single $\mathrm{Co}^{2+}$ ion in a CdTe QD and a single $\mathrm{Mn}^{2+}$ ion in a CdSe QD are presented for the first time. The QD emission decay time is found to be equal in the case of magnetic ion doped and undoped QDs. This indicates 
Table 1 | Tables summarizing semiconductor systems where interaction of excitons and magnetic ion has been confirmed using optical spectroscopy.

a

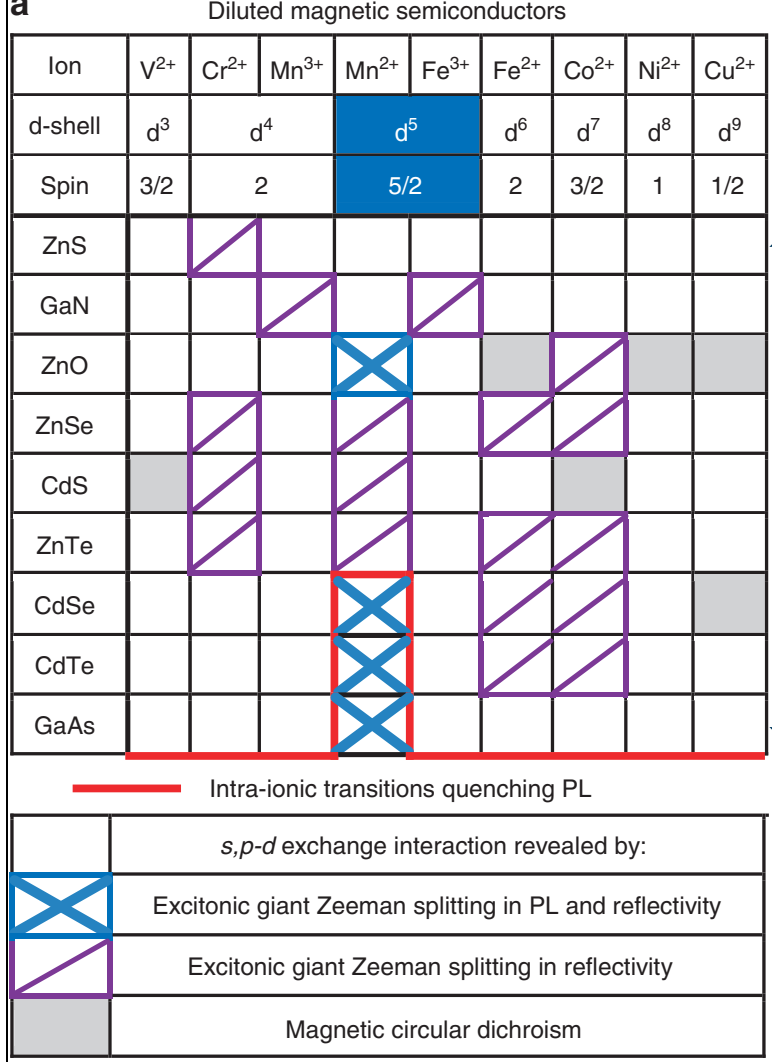

b

\begin{tabular}{|c|c|c|c|c|c|c|c|c|c|}
\hline Atom & V & $\mathrm{Cr}$ & M & In & $\mathrm{F}$ & e & Co & $\mathrm{Ni}$ & $\mathrm{Cu}$ \\
\hline Nuclei spin & $7 / 2$ & 0 & & /2 & ( & 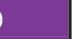 & $7 / 2$ & 0 & $3 / 2$ \\
\hline Ion & $\mathrm{V}^{2+}$ & $\mathrm{Cr}^{2+}$ & $\mathrm{Mn}^{3+}$ & $\mathrm{Mn}^{2+}$ & $\mathrm{Fe}^{3+}$ & $\mathrm{Fe}^{2+}$ & $\mathrm{Co}^{2+}$ & $\mathrm{Ni}^{2+}$ & $\mathrm{Cu}^{2+}$ \\
\hline d-shell & $d^{3}$ & & $d^{4}$ & $d^{2}$ & & $d^{6}$ & $d^{7}$ & $d^{8}$ & $d^{9}$ \\
\hline Electron spin & $3 / 2$ & & 2 & $5 /$ & 2 & 2 & $3 / 2$ & 1 & $1 / 2$ \\
\hline \multicolumn{10}{|l|}{$\mathrm{ZnO} / \mathrm{MgO}$} \\
\hline \multicolumn{10}{|l|}{$\operatorname{lnN} / \mathrm{GaN}$} \\
\hline \multicolumn{10}{|l|}{$\mathrm{CdS} / \mathrm{ZnS}$} \\
\hline CdSe/ZnSe & & & & \begin{tabular}{|l|} 
This \\
work
\end{tabular} & & & & & \\
\hline $\mathrm{CdTe} / \mathrm{ZnTe}$ & & & & {$[10]$} & & & $\begin{array}{l}\text { This } \\
\text { work }\end{array}$ & & \\
\hline \multicolumn{10}{|l|}{ GaAs/AlAs } \\
\hline InAs/GaAs & & & & $\begin{array}{r}{[11]} \\
\mathrm{d} 5+\mathrm{h}\end{array}$ & & & & & \\
\hline \multicolumn{10}{|c|}{-ேー・ } \\
\hline \multicolumn{10}{|c|}{ Anisotropy of magnetic ion: } \\
\hline \multicolumn{10}{|c|}{ Weak (no orbital momentum, or spin $1 / 2$, or strain-free QD) } \\
\hline \multicolumn{10}{|c|}{ Determined by wurtzite structure } \\
\hline \multicolumn{10}{|c|}{ Affected by strain } \\
\hline
\end{tabular}

(a) Diluted magnetic semiconductors (DMS). (b) Quantum dots with single magnetic ions. Vertical order is given by increasing the energy gap, which is anti-correlated with a strength of spin-orbit interaction of a host semiconductor (from weak to strong spin-orbit coupling are systems with anions O, N, S, Se, As and Te). Horizontal order is given by $d$-shell occupancy of magnetic dopants. The area above the red line corresponds to materials exhibiting intra-ionic transitions that quench PL in DMS, but affect only weak PL in QDs with single magnetic ions (as this work shows). A detailed reference list for DMS is given in Supplementary Table 2. QDs with single magnetic ions were reported so far only for two systems CdTe/ZnTe QDs with Mn²+ (ref. 10) and InAs/GaAs QDs with complex of Mn² and bound hole 11 . This work reports on two more systems and indicates that majority of displayed combinations should be useful for optical spectroscopy and for applications.

that quenching of the QD emission is negligible in zerodimensional systems with single magnetic ions. A single manganese in a CdSe QD exhibits the longest spin relaxation time among the single-magnetic-ion-QD systems optically investigated so far. As such, it is clearly a good candidate for a single-spin memory. However, the most promising systems for single-spin memories have still not been tested experimentally: systems with a weak spin-orbit interaction, a wide energy gap and with $d^{5}$ magnetic ions, that is, self-assembled QDs based on oxides, sulphides or nitrides with a single $\mathrm{Mn}^{2+}$ or $\mathrm{Fe}^{3+}$. Further experimental and theoretical studies are needed in order to indicate the optimal QD system for manipulation of a singlemagnetic-ion spin.

\section{Methods}

Growth of samples with self-assembled QDs containing single magnetic ions. Samples are grown on GaAs (100)-oriented substrates using molecular beam epitaxy. After about a 1- $\mu \mathrm{m}$ thick buffer layer of $\mathrm{ZnTe}$ (or $\mathrm{ZnSe}$ ), about three monolayers of CdTe (or CdSe) were grown using atomic layer epitaxy by alternate cycles of $\mathrm{Cd}$ and $\mathrm{Te}$ (or $\mathrm{Se}$ ). In the middle of the $\mathrm{CdTe}$ (or $\mathrm{CdSe}$ ) thin layer, a very small (previously calibrated by giant Zeeman splitting measurements of layers such as $\mathrm{Zn}_{0.997} \mathrm{Co}_{0.003} \mathrm{Te}$ ) amount of magnetic ions: $\mathrm{Co}$ or $\mathrm{Mn}$ are introduced during $3 \mathrm{~s}$ of deposition. Next, the sample is cooled down for $1 \mathrm{~h}$ in $\mathrm{Te}$ (or Se) molecular flux, so at the end sample is covered with amorphous $\mathrm{Te}$ (or Se), which helps the QDs to form $^{34,62}$. Subsequently the sample is heated up to the growth temperature, to sublimate the amorphous Te (or Se). The QDs are covered with a $100 \mathrm{~nm} \mathrm{ZnTe}$ (or ZnSe) cap. No mesas or masks are needed to limit the number of observed QDs.
The emission from single QDs at temperature of $2 \mathrm{~K}$ is collected with a microscope objective assuring a resolution of $0.5 \mu \mathrm{m}$.

Modelling of magneto-PL. A quantitative description of measured QDs magnetoPL spectra (Fig. 2) is provided by a model of the neutral exciton inside a QD with a single magnetic dopant in magnetic field $(B)$ parallel to the growth axis $(z)$. We consider initial and final states of PL transition (Fig. 2e). For the initial state, the model takes into account the energy of an exciton in a nonmagnetic QD (isotropic and anisotropic electron-hole exchange interactions, heavy-light hole mixing, Zeeman effect and diamagnetic shift), energy of ion-electron and ion-hole exchange interactions ( $s, p-d$ interactions), and finally the magnetic ion energy determined by the Zeeman effect and the strain vector. For the final state, we consider only the magnetic ion energy.

The above model is described by the following Hamiltonian $\mathcal{H}_{\mathrm{X}, \mathrm{M}}(B)$ :

$$
\begin{aligned}
\mathcal{H}_{\mathrm{X}, \mathrm{M}}(B)= & \mu_{\mathrm{B}} B\left(g_{\mathrm{e}} S_{\mathrm{e}}^{z}+g_{\mathrm{h}} S_{\mathrm{h}}^{z}\right)+I_{\mathrm{e}} \vec{S}_{\mathrm{e}} \vec{J}+I_{\mathrm{h}} \vec{S}_{\mathrm{h}} \vec{J}-\frac{2 \delta_{0}}{3} S_{\mathrm{e}}^{z} S_{\mathrm{h}}^{z}+\frac{2 \delta_{1}}{3}\left(S_{\mathrm{e}}^{y}\left(S_{\mathrm{h}}^{y}\right)^{3}-S_{\mathrm{e}}^{x}\left(S_{\mathrm{h}}^{x}\right)^{3}\right) \\
& +\Delta_{\mathrm{hh}-\mathrm{hh}}\left(\left(S_{\mathrm{h}}^{x}\right)^{2}-\left(S_{\mathrm{h}}^{y}\right)^{2}\right)+\rho\left(\left(S_{\mathrm{h}}^{x}\right)^{2}+\left(S_{\mathrm{h}}^{y}\right)^{2}\right)+\gamma B^{2}+\mathcal{H}_{\mathrm{M}}, \\
& \mathcal{H}_{\mathrm{M}}=\mu_{\mathrm{B}} B g_{\mathrm{M}} J^{z}+D_{z}\left(J^{z}\right)^{2}+D_{x}\left(J^{x}\right)^{2}+D_{y}\left(J^{y}\right)^{2},
\end{aligned}
$$

where $\mathcal{H}_{\mathrm{M}}$ is the Hamiltonian of the magnetic ion, $g_{\mathrm{e}}, g_{\mathrm{h}}$ and $g_{\mathrm{M}}$ are electron, hole and magnetic ion $g$ factors, $\vec{S}_{e}, \vec{S}_{h}$ and $\vec{J}$ are electron, hole and magnetic ion spin operators, $I_{\mathrm{e}}$ and $I_{\mathrm{h}}$ are the electron-ion and hole-ion exchange constants ${ }^{10,63}, \delta_{0}$ and $\delta_{1}$ are the energies related to isotropic and anisotropic parts of the electronhole exchange interaction ${ }^{64}, \Delta_{\mathrm{lh}-\mathrm{hh}}$ is the heavy-light hole splitting, $\rho$ represents the strength of the valence-band mixing ${ }^{65,66}$ and $\gamma$ is an excitonic diamagnetic shift constant. The parameters $D_{x}, D_{y}, D_{z}$ describe strain-induced zero-field splitting and the anisotropy axis of $\mathrm{Co}^{2+}$ ion spin states. In the case of $\mathrm{Mn}^{2+}$ ion, strain effects ${ }^{67}$ are much weaker and zero-field splitting is neglected. Supplementary Table 1 lists example parameters of the Hamiltonian. These parameters were used for calculation of spectra of CdSe QD with single $\mathrm{Mn}^{2+}$ and CdTe QD with single 
$\mathrm{Co}^{2+}$ presented in Fig. 2 b,d. Parameter $g_{\mathrm{M}}$ for a given ion is from literature data $^{68,69}$. Other parameters were determined from a fit to the data presented in Fig. 2a,c. The oscillator strength of each optical transition is calculated for initial state given by $\mathcal{H}_{\mathrm{X}, \mathrm{M}}$ and final state given by $\mathcal{H}_{\mathrm{M}}$. Line intensity is calculated as a multiplication of oscillator strength and magnetic ion spin state occupancy given by the Boltzmann distribution for an effective temperature $T_{\text {eff. }}$ In this simple model, relaxation of the exciton-ion complex is neglected.

\section{References}

1. Koenraad, P. M. \& Flatté, M. E. Single dopants in semiconductors. Nat. Mater. 10, 91-100 (2011).

2. Awschalom, D. D., Bassett, L. C., Dzurak, A. S., Hu, E. L. \& Petta, J. R. Quantum spintronics: engineering and manipulating atom-like spins in semiconductors. Science 339, 1174-1179 (2013).

3. Fernández-Rossier, J. Single-atom devices: quantum engineering. Nat. Mater. 12, 480-481 (2013)

4. Jelezko, F., Gaebel, T., Popa, I., Gruber, A. \& Wrachtrup, J. Observation of coherent oscillations in a single electron spin. Phys. Rev. Lett. 92, 076401 (2004).

5. Balasubramanian, G. et al. Ultralong spin coherence time in isotopically engineered diamond. Nat. Mater. 8, 383-387 (2009).

6. Dolde, F. et al. Room-temperature entanglement between single defect spins in diamond. Nat. Phys. 9, 139-143 (2013).

7. Yale, C. G. et al. All-optical control of a solid-state spin using coherent dark states. Proc. Natl Acad. Sci. USA 110, 7595-7600 (2013).

8. Weber, J. R. et al. Quantum computing with defects. Proc. Natl Acad. Sci. USA 107, 8513-8518 (2010)

9. Koehl, W. F., Buckley, B. B., Heremans, F. J., Calusine, G. \& Awschalom, D. D. Room temperature coherent control of defect spin qubits in silicon carbide. Nature 479, 84-87 (2011).

10. Besombes, L. et al. Probing the spin state of a single magnetic ion in an individual quantum dot. Phys. Rev. Lett. 93, 207403 (2004).

11. Kudelski, A. et al. Optically probing the fine structure of a single $\mathrm{Mn}$ atom in an InAs quantum dot. Phys. Rev. Lett. 99, 247209 (2007).

12. Goryca, M. et al. Optical manipulation of a single Mn spin in a CdTe-based quantum dot. Phys. Rev. Lett. 103, 087401 (2009).

13. Léger, Y., Besombes, L., Fernández-Rossier, J., Maingault, L. \& Mariette, H. Electrical control of a single $\mathrm{Mn}$ atom in a quantum dot. Phys. Rev. Lett. 97, 107401 (2006)

14. Fernández-Rossier, J. \& Aguado, R. Single-electron transport in electrically tunable nanomagnets. Phys. Rev. Lett. 98, 106805 (2007).

15. Gall, C. L. et al. Optical spin orientation of a single manganese atom in a semiconductor quantum dot using quasiresonant photoexcitation. Phys. Rev. Lett. 102, 127402 (2009).

16. Baudin, E., Benjamin, E., Lematre, A. \& Krebs, O. Optical pumping and a nondestructive readout of a single magnetic impurity spin in an InAs/GaAs quantum dot. Phys. Rev. Lett. 107, 197402 (2011).

17. Besombes, L., Cao, C. L., Jamet, S., Boukari, H. \& Fernández-Rossier, J. Optical control of the spin state of two Mn atoms in a quantum dot. Phys. Rev. B 86, 165306 (2012).

18. Beaulac, R., Schneider, L., Archer, P. I., Bacher, G. \& Gamelin, D. R. Light-induced spontaneous magnetization in doped colloidal quantum dots. Science 325, 973-976 (2009).

19. Kłopotowski, L. et al. Magnetic polaron formation and exciton spin relaxation in single $\mathrm{Cd}_{1-} \mathrm{Mn}_{x} \mathrm{Te}$ quantum dots. Phys. Rev. B 83, 081306 (2011).

20. Andrade, J. A., Aligia, A. A. \& Quinteiro, G. F. Effective Hamiltonian for the impurity-impurity interaction in micropillars in the presence of decoherence. Phys. Rev. B 85, 165421 (2012).

21. Oka, Y. et al. Dynamics of excitonic magnetic polarons in nanostructure diluted magnetic semiconductors. J. Lumin. 83-84, 83-89 (1999).

22. Tang, X. et al. Growth and characterization of CdSe:Mn quantum dots. J. Cryst. Growth 251, 586-590 (2003).

23. Lee, S., Dobrowolska, M. \& Furdyna, J. K. Effect of spin-dependent $\mathrm{Mn}^{2+}$ internal transitions in $\mathrm{CdSe} / \mathrm{Zn}_{1-x} \mathrm{Mn}_{x} \mathrm{Se}$ magnetic semiconductor quantum dot systems. Phys. Rev. B 72, 075320 (2005).

24. Beaulac, R., Archer, P. I., van Rijssel, J., Meijerink, A. \& Gamelin, D. R. Exciton storage by $\mathrm{Mn}^{2+}$ in colloidal $\mathrm{Mn}^{2+}$-doped CdSe quantum dots. Nano Lett. 8, 2949-2953 (2008).

25. Beaulac, R. et al. Spin-polarizable excitonic luminescence in colloidal $\mathrm{Mn}^{2+}$ doped CdSe quantum dots. Nano Lett. 8, 1197-1201 (2008).

26. Zhong, W., Liang, J. \& Yu, J. Systematic study of the interaction of cobalt ions with different-sized CdTe quantum dots. Spectrochim. Acta A Mol. Biomol. Spectrosc. 74, 603-606 (2009)

27. Bussian, D. A. et al. Tunable magnetic exchange interactions in manganesedoped inverted core-shell ZnSe-CdSe nanocrystals. Nat. Mater. 8, 35-40 (2009).

28. Yamamoto, S. Photoluminescence quenching in cobalt doped $\mathrm{ZnO}$ nanocrystals. J. Appl. Phys. 111, 094310 (2012).
29. Pandey, A. et al. Long-lived photoinduced magnetization in copper-doped ZnSe-CdSe core-shell nanocrystals. Nat. Mater. 7, 792-797 (2012).

30. Goryca, M. et al. Brightening of dark excitons in a single CdTe quantum dot containing a single $\mathrm{Mn}^{2+}$ ion. Phys. Rev. B 82, 165323 (2010).

31. Krebs, O., Benjamin, E. \& Lematre, A. Magnetic anisotropy of singly Mn-doped InAs/GaAs quantum dots. Phys. Rev. B 80, 165315 (2009).

32. Le Gall, C., Brunetti, A., Boukari, H. \& Besombes, L. Electron-nuclei spin dynamics in II-VI semiconductor quantum dots. Phys. Rev. B 85, 195312 (2012).

33. Kazimierczuk, T. et al. Magnetophotoluminescence study of intershell exchange interaction in CdTe/ZnTe quantum dots. Phys. Rev. B 84, 165319 (2011).

34. Wojnar, P. et al. Microluminescence from (Cd,Mn)Te magnetic quantum dots containing only a few Mn ions. Phys. Rev. B 75, 155301 (2007).

35. Macfarlane, R. M. Zero field splittings of $t_{2}^{3}$ cubic terms. J. Chem. Phys. 47, 2066-2073 (1967).

36. Koidl, P. Optical absorption of $\mathrm{Co}^{2+}$ in ZnO. Phys. Rev. B 15, 2493 (1977).

37. Pacuski, W. et al. Effect of the $s, p-d$ exchange interaction on the excitons in (Zn,Co)O epilayers. Phys. Rev. B 73, 035214 (2006).

38. Weakliem, H. A. Optical spectra of $\mathrm{Ni}^{2+}, \mathrm{Co}^{2+}$, and $\mathrm{Cu}^{2+}$ in tetrahedral sites in crystals. J. Chem. Phys. 36, 2117-2140 (1962).

39. Baranowski, J. M., Allen, J. W. \& Pearson, G. L. Crystal-field spectra of $3 d^{n}$ impurities in II-VI and III-V compound semiconductors. Phys. Rev. 160, 627-632 (1967).

40. Cibert, J. \& Scalbert, D. in Spin Physics in Semiconductors. Vol. 157 of Springer Series in Solid-State Sciences (ed. Dyakonov, M.) 389-431 (Springer, 2008).

41. Goryca, M. et al. Magnetization dynamics down to a zero field in dilute (Cd,Mn)Te quantum wells. Phys. Rev. Lett. 102, 046408 (2009).

42. Poggio, M., Myers, R. C., Stern, N. P., Gossard, A. C. \& Awschalom, D. D. Structural, electrical, and magneto-optical characterization of paramagnetic GaMnAs quantum wells. Phys. Rev. B 72, 235313 (2005).

43. Myers, R. C. et al. Zero-field optical manipulation of magnetic ions in semiconductors. Nat. Mater. 7, 203-208 (2008).

44. Giriat, W. \& Stankiewicz, J. Photoluminescence in $\mathrm{Mn}_{x} \mathrm{Cd}_{1-x} \mathrm{Se}$ crystals. Phys. Stat. Sol. (a) 59, K79-K80 (1980).

45. Nozik, A. J. Spectroscopy and hot electron relaxation dynamics in semiconductor quantum wells and quantum dots. Annu. Rev. Phys. Chem. 52, 193-231 (2001)

46. Li, X.-Q., Nakayama, H. \& Arakawa, Y. Phonon bottleneck in quantum dots:role of lifetime of the confined optical phonons. Phys. Rev. B 59, 5069-5073 (1999).

47. Morris, D., Perret, N. \& Fafard, S. Carrier energy relaxation by means of Auger processes in InAs/GaAs self-assembled quantum dots. Appl. Phys. Lett. 75, 3593-3595 (1999).

48. Ferreira, R. \& Bastard, G. Phonon-assisted capture and intradot Auger relaxation in quantum dots. Appl. Phys. Lett. 74, 2818-2820 (1999).

49. Cohn, A. W., Schimpf, A. M., Gunthardt, C. E. \& Gamelin, D. R. Sizedependent trap-assisted Auger recombination in semiconductor nanocrystals. Nano Lett. 13, 1810-1815 (2013).

50. Nawrocki, M., Rubo, Y. G., Lascaray, J. P. \& Coquillat, D. Suppression of the Auger recombination due to spin polarization of excess carriers and $\mathrm{Mn}^{2+}$ ions in the semimagnetic semiconductor $\mathrm{Cd}_{0.95} \mathrm{Mn}_{0.05} \mathrm{~S}$. Phys. Rev. B 52, R2241-R2244 (1995)

51. Kim, C. S. et al. CdSe quantum dots in a $\mathrm{Zn}_{1-x} \mathrm{Mn}_{x}$ Se matrix: new effects due to the presence of Mn. J. Cryst. Growth 214-215, 395-399 (2000).

52. Chernenko, A. V. et al. Auger recombination of excitons in semimagnetic quantum dot structure in a magnetic field. Phys. Rev. B 72, 045302 (2005).

53. Jamet, S., Boukari, H. \& Besombes, L. Spin dynamics of a Mn atom in a semiconductor quantum dot under resonant optical excitation. Phys. Rev. B 87, 245306 (2013).

54. Kusrayev, Y. G. et al. Optical orientation and alignment of excitons in self-assembled CdSe/ZnSe quantum dots: The role of excited states. Phys. Rev. B 72, 155301 (2005).

55. Blume, M. \& Orbach, R. Spin-lattice relaxation of $S$-state ions: $\mathrm{Mn}^{2+}$ in a cubic environment. Phys. Rev 127, 1587-1592 (1962).

56. Cao, C. L., Besombes, L. \& Fernández-Rossier, J. Spin-phonon coupling in single Mn-doped CdTe quantum dot. Phys. Rev. B 84, 205305 (2011)

57. Wang, X.-F., Wu, S.-Y., Li, L.-L. \& Zhang, S.-X. Investigations of the spin hamiltonian parameters for the cubic $\mathrm{Mn}^{2+}$ centers in $\mathrm{ZnX}(\mathrm{X}=\mathrm{S}, \mathrm{Se}, \mathrm{Te})$ and CdTe. Can. J. Phys. 88, 301-306 (2010).

58. Lambe, J. \& Kikuchi, C. Paramagnetic resonance of CdTe: Mn and CdS: Mn. Phys. Rev 119, 1256-1260 (1960).

59. Deville, A., Gaillard, B., Blanchard, C. \& Landi, A. Spin-lattice relaxation of $\mathrm{Fe}^{3+}$ in ZnS; experimental evidence for optical phonons. J. Phys. 40, 1173-1178 (1979).

60. Solomon, P. R. Relaxation of $\mathrm{Mn}^{2+}$ and $\mathrm{Fe}^{3+}$ ions in magnesium oxide. Phys. Rev. 152, 452-466 (1966).

61. Ochsenbein, S. T. \& Gamelin, D. R. Quantum oscillations in magnetically doped colloidal nanocrystals. Nat. Nanotech. 6, 112 (2010). 
62. Tinjod, F., Gilles, B., Moehl, S., Kheng, K. \& Mariette, H. II-VI quantum dot formation induced by surface energy change of a strained layer. Appl. Phys. Lett. 82, 4340-4342 (2003).

63. Gaj, J. A. \& Kossut, J. in Introduction to the Physics of Diluted Magnetic Semiconductors Vol. 144 Springer Series in Materials Science (eds Gaj, J. A. \& Kossut, J.) 1-36 (Springer, 2010).

64. Bayer, M. et al. Fine structure of neutral and charged excitons in self-assembled In(Ga)As/(Al)GaAs quantum dots. Phys. Rev. B 65, 195315 (2002).

65. Léger, Y., Besombes, L., Maingault, L. \& Mariette, H. Valence-band mixing in neutral, charged, and Mn-doped self-assembled quantum dots. Phys. Rev. B 76, 045331 (2007)

66. Koudinov, A. V., Akimov, I. A., Kusrayev, Y. G. \& Henneberger, F. Optical and magnetic anisotropies of the hole states in Stranski-Krastanov quantum dots. Phys. Rev. B 70, 241305 (2004).

67. Qazzaz, M. et al. Electron paramagnetic resonance of $\mathrm{Mn}^{2+}$ in strained-layer semiconductor superlattices. Solid State Commun. 96, 405-409 (1995).

68. Ham, F. S., Ludwig, G. W., Watkins, G. D. \& Woodbury, H. H. Spin hamiltonian of $\mathrm{Co}^{2+}$. Phys. Rev. Lett. 5, 468-470 (1960).

69. Title, R. S. Electron paramagnetic resonance detection of lattice distortion in $\mathrm{Mn}^{+}{ }^{+}$-doped II-VI compounds. Phys. Rev. 131, 2503-2504 (1963).

\section{Acknowledgements}

We acknowledge helpful discussions with Ł. Cywiński, T. Dietl, T. Kazimierczuk, Ł. Kłopotowski and P. Wojnar. This work was partially supported by the Polish National Science Centre under decisions DEC-2011/01/B/ST3/02406, DEC-2011/02/A/ST3/00131, DEC-2012/05/N/ST3/03209 and DEC-2013/09/B/ST3/02603, by the Polish National Centre for Research and Development project LIDER, by the Foundation for Polish Science and by Polish Ministry of Science and Higher Education as research grants
'Diamentowy Grant'. The project was carried out with the use of CePT, CeZaMat and NLTK infrastructures financed by the European Union-the European Regional Development Fund within the Operational Programme 'Innovative economy' for 2007-2013. The work was also supported by the EC through the FunDMS Advanced Grant of the European Research Council (FP7 'Ideas') under grant agreement no. 227690 and by the European Union Seventh Framework Programme (FP7/2007-2013) under grant agreement no. 316244 .

\section{Author contributions}

J.K., M.P., K.G., J.-G.R., E.J. and W.P. grew and characterized samples. J.K., T.S., M.K. M.G., A.G., P.K. and W.P. performed magneto-optical experiments, data analysis and modelling. T.S., A.B. and M.G. performed single-spin relaxation measurements. T.S., J.K., J.S., M.N., A.G. and W.P. prepared the manuscript in consultation with all authors.

\section{Additional information}

Supplementary Information accompanies this paper at http://www.nature.com/ naturecommunications

Competing financial interests: The authors declare no competing financial interests.

Reprints and permission information is available online at http://npg.nature.com/ reprintsandpermissions/

How to cite this article: Kobak, J. et al. Designing quantum dots for solotronics. Nat. Commun. 5:3191 doi: 10.1038/ncomms4191 (2014).

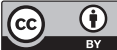

This article is licensed under a Creative Commons Attribution 3.0 Unported Licence. To view a copy of this licence visit http:// creativecommons.org/licenses/by/3.0/. 